\title{
Improving FEM crash simulation accuracy through local thickness estimation based on CAD data
}

\author{
Vânio Ferreira, Luís Paulo Santos, * \\ Markus Franzen, Omar O. Ghouati, ${ }^{\dagger}$ \\ Ricardo Simoes ${ }^{\ddagger}$
}

February 5, 2014

\begin{abstract}
In this paper, we present a method for estimating local thickness distribution in finite element models, applied to injection molded and cast engineering parts. This method features considerable improved performance compared to two previously proposed approaches, and has been validated against thickness measured by different human operators. We also demonstrate that the use of this method for assigning a distribution of local thickness in FEM crash simulations results in a much more accurate prediction of the real part performance, thus increasing the benefits of computer simulations in engineering design by enabling zero-prototyping and thus reducing product development costs. The simulation results have been compared to experimental tests, evidencing the advantage of the proposed method. Thus, the proposed approach to consider local thickness distribution in FEM crash simulations has high potential on the product development process of complex and highly demanding injection molded and casted parts and is currently being used by Ford Motor Company.
\end{abstract}

\section{Introduction}

The automotive industry is continuously faced with a rising number of challenges, including, among others, requirements to reduce vehicle weight - motivated by the need to reduce $\mathrm{CO} 2$ emissions - and to increase protection and safety for occupants and pedestrians. These challenges take a strong influence on the development process and make it necessary to fully utilize deployed materials as efficiently as possible. Additionally, product development has to be economical with respect to development time and costs. To

\footnotetext{
${ }^{*}$ CCTC, Universidade do Minho, Braga, Portugal, vaniomiguel@gmail.com and psantos@di.uminho.pt

${ }^{\dagger}$ Ford Research \& Advanced Engineering Europe, 52072 Aachen, Germany, [mfranze6,oghouati]@ford.com

${ }^{\ddagger}$ Institute of Cávado and Ave, Barcelos, Portugal and Institute for Polymers and Composites IPC/I3N, University of Minho, Guimaraes, Portugal, rsimoes@dep.uminho.pt
} 
comply with these ever increasing challenges car manufacturers are intensifying the use of Computer-Aided Engineering (CAE) tools for the various stages of the development process. One such stages is vehicle crash simulation, a mathematically very complex event, which led to the proposal of different representation and analysis approaches including Morlet wavelets [7], the Levenberg-Marquardt optimization algorithm [10], non linear autoregressive models [11] and adaptive neurofuzzy inference systems [17], among others. Finite Element Methods (FEM) have been a standard tool for a long time, particularly for modeling and simulating thermoplastic parts under service conditions $[12,16]$. However, these approaches can only be successful if the numerical methods are capable and have a high confidence level.

Within the area of car crash simulation, thermoplastic parts are particularly challenging due to the intrinsic complex behavior of those materials. However, as plastics are pervasive in many different automotive applications, including those with very demanding specifications, it becomes vital to find adequate methods for modeling and simulating those parts under service conditions.

Due to the fact that most thermoplastic parts used in vehicles are injection molded, the actual local thicknesses can vary significantly throughout a part (see figure 1), especially near edges, joints, ribs and mold drafts. This has an effect on the accuracy of the deformation and fracture behavior prediction. However, local thickness distribution is

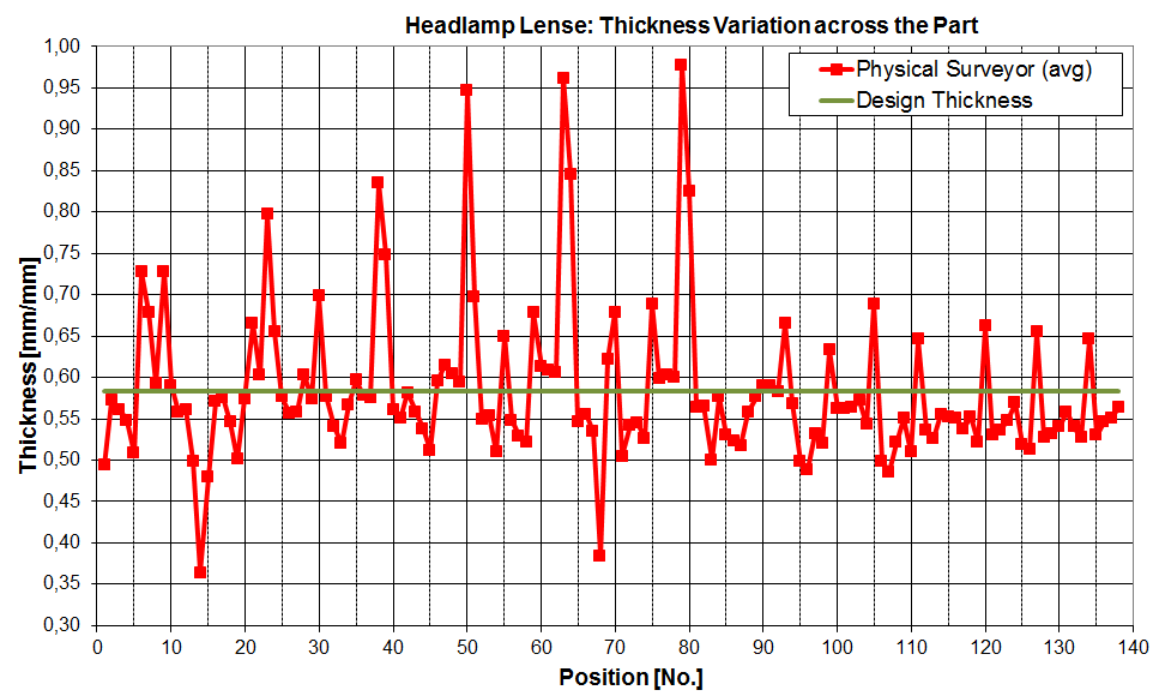

Figure 1: Normalized thickness variation across a headlamp depicted as an average of the physical measurements performed by two surveyors (see section 4.1 for an explanation on the physical measurements). Notice how it locally deviates from the average design thickness, typically used in car crash simulations.

not explicitly available in FEM midplane meshes. Therefore current state of the art within vehicle crash simulations is to resort to two alternative approaches: 
i) for simple parts, which are not highly loaded in crash scenarios, a constant design thickness is often used;

ii) for more complex and/or highly stressed parts, manual segmentation is applied to use different constant thicknesses in feature areas, which is very time consuming and not applicable within automotive series developments.

These thickness values are based on digital manual CAD measurements due to the fact that physical parts are not available at this stage within the development process. Both approaches result in deviations between simulation and physical tests in the area of deformation behavior, force-deflection characteristics and especially fracture prediction due to simplifications in the thickness range. Thus it is absolutely vital that elements' thicknesses are properly assigned. This local thickness information exists implicitly in the full part geometry in Computer Aided Design (CAD) files. Automated and precise ways to extract that local thickness distribution from the 3D CAD files to 2D midplane elements used within automotive series development simulations are therefore required to avoid limiting the precision, and thus the potential benefit, of crash simulations. The goal is to allow for zero-prototyping, such that physical parts are only produced once the development process is finished. Vehicle development engineers work only with CAD data, upon which CAE models are built and simulations run. Results from these simulations are applied to the CAD models to comply with all boundary conditions and legislation. No cost and time expensive re-engineering and re-tooling should be required after the production of physical parts, which places high accuracy requirements on crash simulations.

In previous work two thickness estimation techniques were proposed [5], based on ray tracing (RT) and nearest neighbor 3D range searches (NN), which allow tagging each midplane mesh element with its associated local thickness (see figure 2), thus empowering accurate vehicle crash simulations. It was shown that both techniques complement each other: if each algorithm's best estimate can be selected for each element of the midplane mesh, then accuracy is improved. Identifying the best estimate for each element is not evident, however, due to the complexity of real world parts. Real world geometries and midplane meshes have lots of details and particular configurations that make it very difficult to establish which is the best estimate - particularly, the real local thicknesses are not known, since this is exactly the quantity that is being measured. Additionally, there are some pathological geometric and midplane mesh configurations which hinder both algorithms from computing any thickness estimate at all. These elements are then tagged as incorrect and must be processed manually by a human operator. Thus, the previously proposed method is unable to provide the thickness distribution information that is required in a practical industrial context.

The present paper extends the above cited approach, enabling accurate thickness estimation for all midplane mesh elements. Its contributions are four-fold:

- a criterion for deciding whether RT and NN thickness estimates for a given element are accurate based on their relative values; 


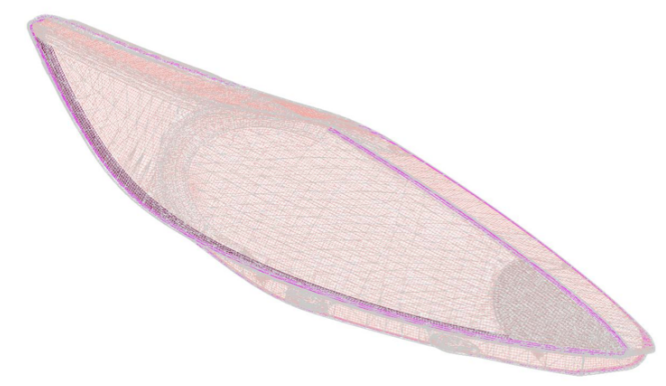

(a) Geometry and midplane mesh.

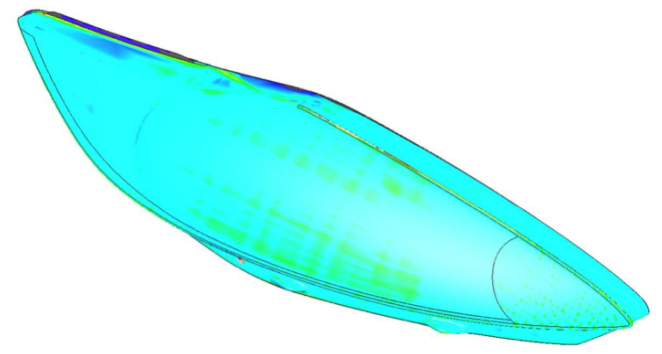

(b) Estimated Thickness.

Figure 2: (a) presents an automotive headlamp lens geometry (grey) and the respective crash simulation midplane mesh (red); ideally, the midplane mesh runs inside the part's surface, but in reality it might extend outside due to approximations. (b) presents, for each element of the midplane mesh, the estimated normalized thickness as a pseudo color map using the thickness estimation method proposed on this paper.

- a technique, based on propagation from neighboring elements, for estimating an element's thickness when both RT and NN algorithms fail to compute an accurate approximation;

- a comprehensive pipeline for completely automated estimation of local thicknesses, given a part's surface geometry description and the corresponding midplane mesh;

- a systematic analysis of the proposed pipeline accuracy, including a validation against thickness measured by different operators and comparisons of FEM crash simulations with and without local thickness estimates against related dynamic physical component tests using a pedestrian protection load case.

Thus, the main goal of this work is to enable a fully automated approach to extract local thickness information from a full 3D mesh, and assign an appropriate value of local thickness to every element in the associated 2D midplane mesh, dispensing with using a unique design thickness for the entire part (a gross approximation) or performing manual segmentation (a time-consuming and error-prone operation). Midplane meshes are state of the art within vehicle development because they deliver good quality results in a timely manner. Solid modelling would be an alternative but due to the huge amount of computer capacity needed to handle these big models and significantly increased calculation times, they are currently seldom used for vehicle development.The presented results demonstrate that not only is local thickness accurately estimated, but also that these enhanced estimations enable more accurate prediction of the crash behavior of engineering parts. This is a significant improvement regarding crash simulation quality, allowing for zero-prototyping and resulting in reduced re-testing of physical components, saving time, human resources and money. Therefore this approach constitutes a significant contribution and will have a substantial impact on engineering practice, particularly within the 
automotive industry, where Ford Motor Company is already using this approach.

It is important to note that all part thickness values presented in this paper are normalized for confidentiality reasons. Normalization is performed by dividing all thickness values by the part's maximum thickness. Thus, the thicknesses listed do not represent the real thickness of the case-study parts and their units are $\mathrm{mm} / \mathrm{mm}$.

The next section presents a thickness estimation method proposed by Ferreira et al. [5] and discusses its limitations. Section 3 describes the newly proposed approach, whereas section 4 presents and analyzes experimental results. The paper closes with some concluding remarks.

\section{Thickness Estimation Fundamentals}

This section describes previous work published by Ferreira et al. [5], which proposes two methods for local thickness estimation from a part's geometric description. Original results presented in this paper build upon these methods.

One broadly accepted definition of the local thickness of a three dimensional object with respect to any given point $p$ on the object's surface is based on its Medial Axis Transform (MAT) [9]. Since each such point $p$ has a unique corresponding point $m$ on the medial axis, local thickness at $p$ can be defined as the radius of the sphere centered at $m$ and tangent to $p$. Since the MAT is a computationally very expensive process Gal et al. [6] propose the Local Diameter Shape Function for any point $p$ on the surface of the object, which is based on tracing a large number of rays in a cone around the direction opposite to the surface's normal at $p$ and finding the distance to the next surface intersection point along each ray. The local thickness is then given by the average length of the rays after discarding outliers.

The problem addressed within this paper is different in the sense that one wishes to estimate local thickness around the centroids of the midplane mesh and not for points on the surface of the object. The generation of the midplane mesh itself is not addressed here, since this is already used in FEM crash simulations and is made available by product development engineers using the CAD system. The two basis algorithms are however still based on the notion of ray tracing and/or cones of directions, but centered on the midplane mesh elements' centroids and distributed around this mesh normals for more details see next subsection. In this context local thickness at the centroid of each midplane mesh element is broadly defined as the sum of the distances from the centroid to some surface point on each side of the element.

When correctly generated the midplane mesh runs inside the part's surface and parallel to it. However, creating midplane meshes is not trivial. There are several techniques and applications for mesh generation and mesh refinement, but there is still ongoing research on finding automated (or semi-automated) procedures that enable obtaining 
meshes of adequate quality for simulation purposes $[2,8,13]$. In practice, midplane meshes currently used for FEM are coarse approximations of the part's surface geometry. Consequently, details on the original geometry might be lost and the midplane mesh often runs partially outside the part's surface. These representation inaccuracies lead to thickness estimation errors as described in the next subsections.

\subsection{Algorithms}

The broad definition of thickness given above lead to two alternative thickness estimation algorithms [5], both optimized by resorting to a kd-tree to spatially sort the surface's geometric primitives:

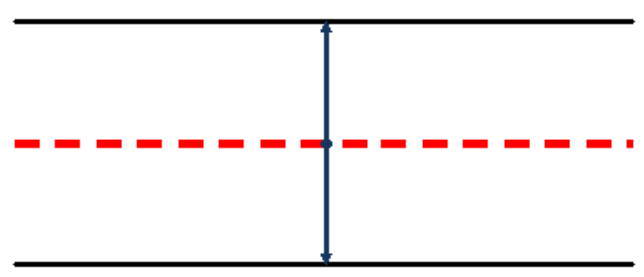

(a) Ray tracing.

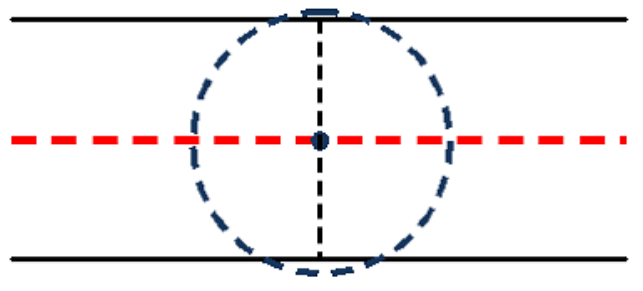

(b) Nearest neighbor.

Figure 3: Algorithms for thickness estimation. Solid black lines represent the part's geometry, the red dashed line represents the midplane mesh and the dashed circle represents the nearest neighbor search domain.

Ray Tracing (RT) - a ray is shot for each side of the midplane mesh element, with origin on the element's centroid and direction equal to the element's normal [1, 15]. Ideally, each of these rays intersects the part's geometry; the sum of both intersections' distances is taken as an estimate of the part's local thickness (see figure $3(\mathrm{a}))$.

Nearest Neighbor (NN) - a search over the part's surface geometry locates which point is nearer to the midplane mesh element centroid [3]. Figure 3(b) illustrates the algorithm; the dashed circle represents the search domain, which grows until the search algorithm returns a valid point on the part's surface. Actually, two such searches are performed to locate two points, each on a different side of the midplane mesh element. The sum of the distances from the element's centroid and these two points is taken as an estimate of the part's local thickness.

\subsection{Limitations and Advantages}

For most cases both algorithms return similar thickness estimates. However, real automative parts and models include complex geometric configurations and incorrect mid- 
plane mesh approximations where either one or both algorithms fail to find robust estimates.

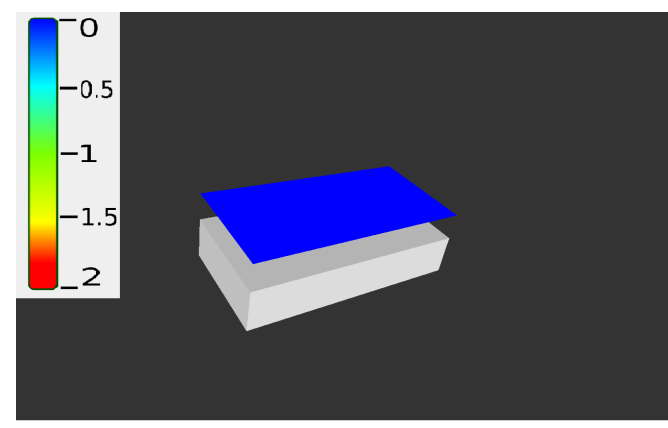

(a) Ray tracing - midplane mesh outside the volume.

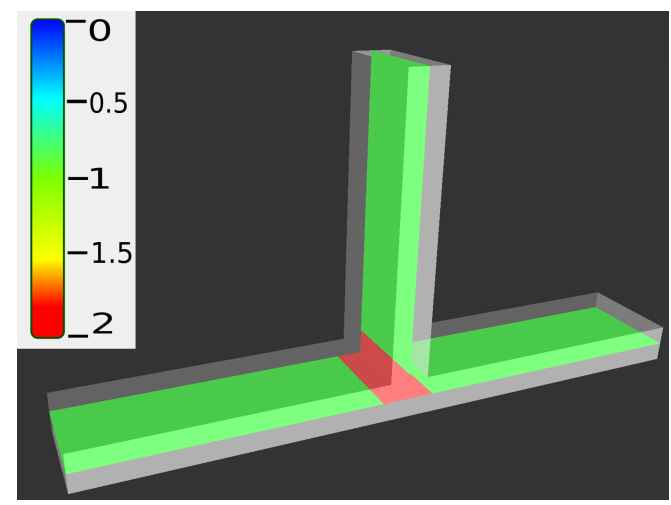

(c) Ray tracing - ribs.

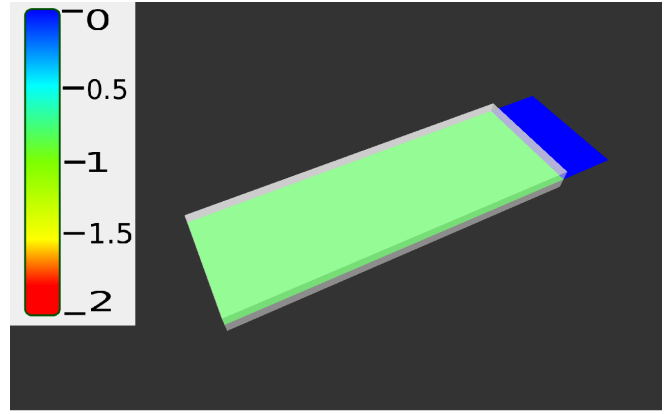

(b) Ray tracing - midplane mesh outside the volume.

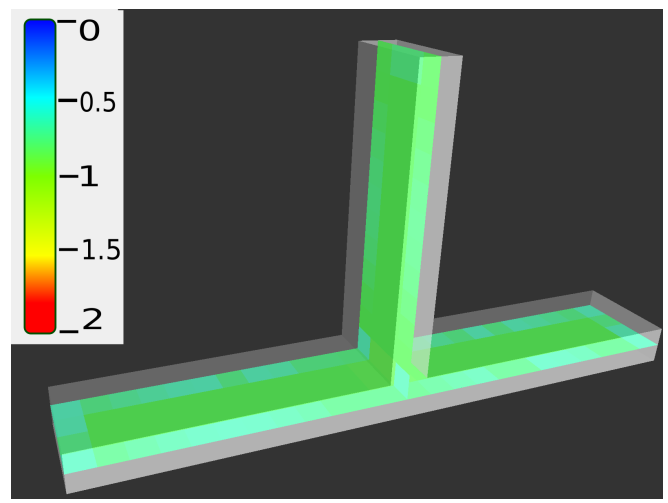

(d) Nearest neighbor - ribs.

Figure 4: Thickness estimation with inaccurate midplane meshes and ribs - pseudo color maps (normalized).

A systematic analysis of the results obtained for a range of parts with various characteristics allowed identifying the following cases where the proposed algorithms are unable to accurately estimate thickness [5]:

1. When the midplane mesh runs outside the part's surface both algorithms fail to find valid points on both sides of the mesh and, consequently, fail to estimate thickness; see figures 4(a) and 4(b).

2. The ray tracing approach fails when the midplane mesh element's centroid is aligned with a rib; see figure 4(c). Rays, which are shot along the element's normal, will run inside the part, finding an intersection at distant points of the part's surface and overestimating thickness. The NN algorithm will still be able to find nearest points near the rib's junction with the part's surface, thus avoiding large thickness estimation errors and clearly outperforming ray tracing; see figure $4(d)$. 
3. The nearest neighbor approach underestimates thickness for elements that are close to the mesh boundaries; see figure 4(d). The spatial search will find the nearest points on the part's lateral surface, rather than on the longitudinal surfaces; the thickness estimate is thus smoothed and diverges from its actual value, suggesting a round edge. The RT algorithm does not suffer from this problem, given that it searches only along the midplane element's normal; see figure 4(c).

Case 1 can be detected during thickness estimation if there is a way of determining whether a midplane mesh element's centroid is contained within the part's volume, as defined by its closed surface. This is a generalization of the well-known point in polygon problem and can be solved by resorting to ray tracing: if a ray is shot from a given point along any direction, that point is inside the closed surface if it intersects the surface an odd number of times; else it is outside the closed surface [14]. Thus, if each of the rays shot by the RT algorithm intersects the surface an odd number of times, then the centroid is inside the part. If both rays intersect the surface an even number of times, then the centroid is outside the surface. In this latter case, and if at least one of the rays intersects the surface more than zero times, the side of the centroid whose ray reported the closest intersection is selected as the one closest to the surface and thickness is estimated as the difference between the two closest intersections of that ray. Two different cases are thus identified: i) the midplane mesh runs outside the part's volume but still encompasses it as in figure 4(a), and ii) the midplane mesh is partially outside the part's volume and does not encompass it as in figure 4(b). Case i) can still be solved by ray tracing, whereas case ii) can not and requires tagging that mesh element as incorrect.

Cases 2 and 3 would be easily solved if the most accurate estimate, among RT and NN, could be identified on a per element basis. Additionally, in order to limit the divergence occurring near the midplane mesh boundaries with the NN algorithm (case 3 above), a limitation can be imposed on the maximum acceptable angle between the element's normal and the direction defined by the element's centroid and the surface nearest point. By limiting this angle it is expected that the part's lateral surface is rejected as a nearest neighbor, thus forcing the algorithm to expand its search onto regions of the surface that are farther away from the mid-plane element. Ferreira et al. [5] show that a limit of $80^{\circ}$ more than halves estimation errors near the mesh boundaries over a wide range of parts.

Summarizing, for most midplane mesh elements NN and RT return very similar thickness estimates and either can be used without impacting on the overall thickness estimation accuracy. Near ribs or near mesh boundaries one of the algorithms fails (cases 2 and 3 ), while the other still accurately estimates thickness: RT and NN complement each other on these cases. Detecting which algorithm returns the most accurate estimate would allow correctly handling these elements, but this is a non trivial problem given the complexity of real parts' geometric configurations. Finally, when the midplane mesh is not contained within the volume defined by the parts' surface both algorithms will fail to find an estimate. Ray tracing can be used to detect such elements by resorting to 
a generalization of the point in polygon algorithm. Furthermore, if the midplane mesh encompasses the part's surface, RT can still generate an estimate of the local thickness. Section 3 proposes a methodology to handle all these cases.

\section{Improving Thickness Estimation Accuracy}

This section proposes a new methodology for generating improved thickness estimates for all midplane mesh elements. It uses the above described ray tracing and nearest neighbor algorithms and resorts to thickness propagation among neighboring elements when an accurate estimate can not be found for a given element. The proposed methodology builds upon the following premises:

- in the presence of well behaved midplane mesh elements both algorithms return similar thickness estimates; if these estimates diverge significantly, then one of the cases described on the previous section applies;

- midplane elements that stand completely outside the part's volume as defined by its closed-surface can not be handled by RT or NN; RT can however be used to identify such elements;

- since thermoplastic parts are injection molded, thickness is expected to change smoothly across a part's surface, even though thickness can change significantly across the whole surface; the fact that thickness is most often locally smooth enables propagation methods, where an element's thickness can be estimated as a function of its neighbors' thicknesses.

The proposed pipeline includes two main stages: stage 1 locally estimates thickness and classifies these estimates as either correct or incorrect, whereas stage 2 handles incorrect estimates.

\subsection{Identifying and Classifying Incorrect Estimates}

The first step of thickness estimation entails generating initial estimates by resorting to the two above described algorithms and then classifying these estimates as either correct or incorrect. This classification is based on whether or not the element's centroid is placed within the part's volume and on an eventual divergence between the RT and NN thickness estimates. Elements for which no correct thickness estimate could be generated are further classified onto three different types of incorrectness, referred to as types A, $\mathrm{B}$ and $\mathrm{C}$. Incorrect elements are inserted on an ordered queue, sorted by the type of incorrectness. A complete description of this first stage is given below and illustrated diagrammatically in figure 5 .

Stage 1.1 entails estimating an element's thickness with the ray tracing algorithm and detecting whether or not the element lies within the part's volume. If it does, then it 


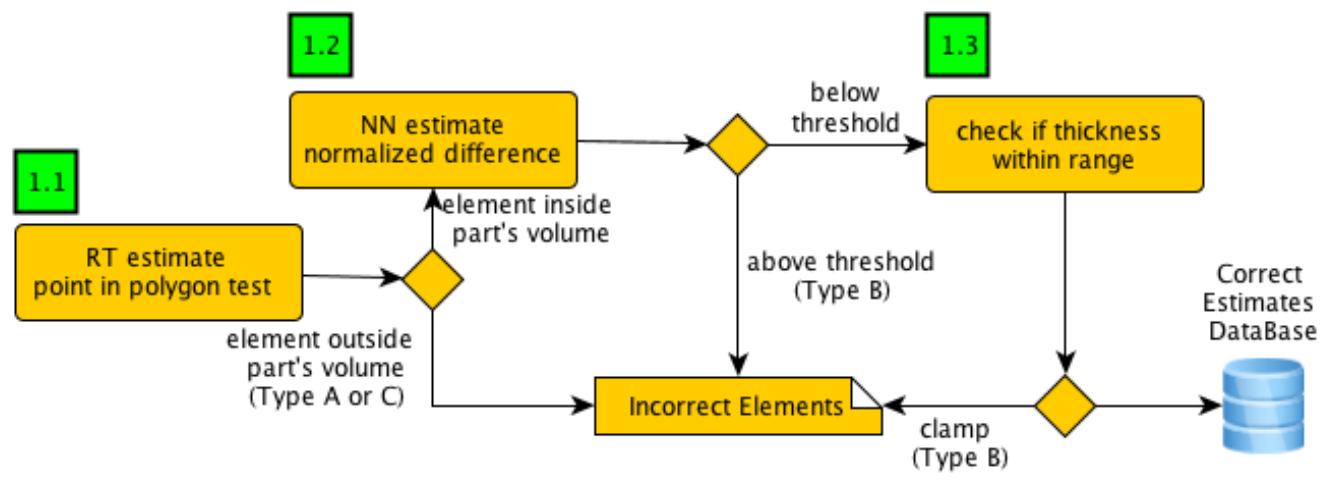

Figure 5: Stage 1 - Identifying and classifying incorrect thickness estimates.

proceeds to stage 1.2, otherwise it will be enqueued as an incorrect element. Elements that lie outside the part's volume might still encompass the part's surface; this being the case, they are classified as being of type A, otherwise they are classified as type $\mathrm{C}$ (no thickness estimate whatsoever is generated for type $\mathrm{C}$ elements). For type A elements, $\mathrm{RT}$ is still able to generate a thickness estimate and this estimate is also stored on the queue. However, the accuracy of this estimate depends on the angle formed by the ray direction and the normal of the part's surface at the intersection point. If this angle is large, then the ray will traverse the part obliquely and overestimates thickness. Type A elements are thus inserted on the ordered queue of incorrect elements sorted by the above described angle; elements with smaller angles will be processed first by stage 2 of the pipeline, decreasing the probability of propagating largely overestimated thicknesses.

Informed by the fact that both $\mathrm{NN}$ and RT return similar estimates over those regions where the midplane mesh is an accurate representation of the part's surface, stage 1.2 computes the element's thickness using the NN algorithm and compares it with the RT estimate. To assess whether or not these two estimates diverge significantly the normalized difference, $\Delta_{i}$, is computed according to equation 1

$$
\Delta_{i}=\frac{\left|T_{i}^{R T}-T_{i}^{N N}\right|}{\max \left(T_{i}^{R T}, T_{i}^{N N}\right)}
$$

where $T_{i}^{R T}$ and $T_{i}^{N N}$ are the ray tracing (RT) and nearest neighbor (NN) thickness estimates for element $i$. Elements exhibiting $\Delta_{i}>\epsilon$ are enqueued as type B incorrect elements together with the respective $\mathrm{NN}$ estimate. The optimum value for the error threshold $\epsilon$ was experimentally determined to be 0.2 , based on an ensemble of engineering parts.

Stage 1.3 detects false positives resorting to user supplied minimum and maximum acceptable thicknesses - these values are known by the parts' designers. If an element 
considered correct by stage 1.2 has a thickness estimate $T_{i}$ that falls outside the interval defined by these minimum and maximum values, then its value is set to either the minimum or maximum, respectively, and the element is enqueued as type B. Finally, thickness estimates that successfully passed the three verification stages are stored on the correct estimates database.

\subsection{Propagation of Thickness Estimates}

At the end of stage 1 the ordered queue contains all those elements for which an accurate thickness estimate could not be found. These will be handled by propagating thickness estimates from neighboring elements which have been stored on the correct estimates database. Propagation is expected to give accurate results because thickness varies smoothly across an injection molded part.

Two elements are considered neighbors if they share at least one vertex and if the angle formed by their normals is below some given threshold; a value of 25 degrees was experimentally determined to give good results in the vast majority of cases. Taking orientation into account for defining neighborhood is fundamental to avoid propagating thickness among elements that belong to geometrically different regions of a part, such as from a rib to the part's main surface. However, as described below, in some cases the concept of neighborhood has to be relaxed and propagation is allowed among connected elements, that is elements that share at least one vertex.

The proposed algorithm (see algorithm 1) applies four different thickness correction criteria $(\mathrm{C} 1$ to $\mathrm{C} 4)$, one at a time, for the totality of elements stored in the incorrect elements queue. Thus, for each criterion, the ordered queue of incorrect elements is sequentially traversed. For all criteria, except C3, the current incorrect element's neighborhood is looked up in the correct estimates database; if the criterion specific conditions are satisfied, the element's thickness is corrected and it is placed in the correct estimates database. The element is then removed from the incorrect elements' queue and processing restarts from the queue's head and criterion $\mathrm{C} 1$. The reason for restarting the algorithm is that, since there are more correct elements, propagation can now be successful for incorrect elements where it failed before.

The four criteria are progressively less restrictive on the conditions imposed to be successfully applied. The incorrect elements are also stored on the queue ordered by the type of error: type A meaning that a ray tracing estimate is available for elements placed outside the surface's volume, type B meaning that the difference between the RT and NN estimates is significant, and type $\mathrm{C}$ when no thickness estimate is available at all. The combination of these two orders, criteria and error type, is crucial to ensure maximum accuracy on the final result.

The criteria are as follows:

C1 - if all direct neighbors of the current element are tagged as correct, then assign to 


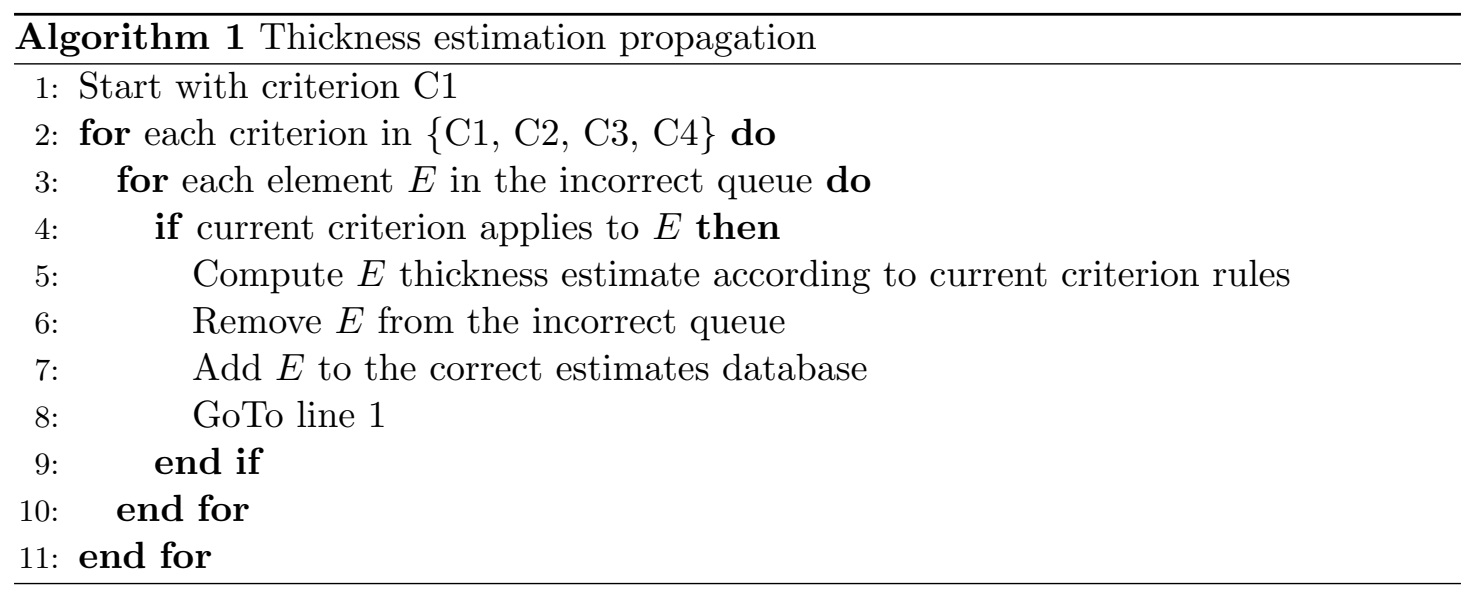

the element their average thickness;

C2 - if at least one direct neighbor of the current element is tagged as correct, then assign to the element the average thickness of all correct direct neighbors;

C3 - if the current element was tagged as type A, i.e., it lies outside the part's volume but its thickness was still estimated by ray tracing, and this estimate is above the minimum thickness and below the maximum, then assign it the estimated thickness;

C4 - assign to the current element the average thickness of all the correct elements with whom it shares at least one vertex, i.e., relax the neighborhood constraint by ignoring relative orientation.

\section{Results}

In order to assess the effectiveness of the proposed approach, thickness estimations obtained with the improved method are compared with:

i) results obtained with the method described in [5];

ii) thickness values obtained by visual inspection of the parts' CAD files;

iii) thickness measurements obtained by physical inspection of the part.

Subsection 4.1 describes the experimental methodologies used; subsection 4.2 compares the proposed approach with that proposed in [5], while subsection 4.3 compares thickness estimates with thickness measurements obtained by physical inspections. Finally, subsection 4.4 compares crash simulation results obtained with the nominal thickness standard procedure with crash simulation results obtained with the proposed thickness estimates; these simulations results are further compared with dynamical physical component tests performed at Ford Motor Company. 


\subsection{Experimental Methodology}

The thickness estimation method proposed in [5], and the improved method proposed on this paper, were independently run over the CAD models of a set of parts identified by Ford Motor Company as representative of the geometries typically found in automotive engineering components. In order to assess the accuracy of the obtained estimates, the values obtained with both methods are compared with thickness values obtained by:

visual inspection - thickness values are manually calculated from the CAD model, by identifying, for each element of the midplane mesh, the points on the surfaces that define the local thickness, according to the operator's best judgement, and measuring the distance between those points;

physical inspection - thickness measurements are physically obtained from real world parts. A production part was cut along regularly distanced cross sections and thickness was measured with a digital gauge at a set of points defined along the cut edges. The midplane mesh element related to each measurement point was identified to allow direct comparisons. Physical measurements were performed by two independent human surveyors - both experienced operators - and averaged in order to reduce any bias associated with the measurement process.

Quantitative comparisons of the quality of the estimates with respect to the baselines (given by visual inspection or physical inspection) will be based on the Root Mean Square Error (RMSE), computed according to equation 2. RMSE takes the square of the individual differences, also called residuals, between the estimated $\left(\tilde{T}_{i}\right)$ and the reference thickness $\left(T_{i}\right)$ at each element $i$ of the mid-plane mesh and aggregates them onto a single metric that has predictive power and is perceived as a good measure of accuracy

[4]. The lower the RMSE the better the thickness estimates produced by the associated algorithm. RMSE heavily weights outliers (i.e., particularly bad local estimates) due to the squaring of the residuals, whereas small residuals are attributed very small weights; it is felt, however, that for Finite Element Analysis of structural properties outliers can strongly affect the simulation result, thus this is a desirable property.

$$
R M S E=\sqrt{\frac{\sum_{i=1}^{N}\left(\tilde{T}_{i}-T_{i}\right)^{2}}{N}}
$$

As stated in section 1, all part thickness values presented in this paper are normalized, by diving all thicknesses by the part's maximum thickness. For the Door Handle and B-Pillar Trim parts (figures 6, 7, 8 and table 1) the maximum thickness was taken from the visual inspection measurements, which means that thickness estimates above 1 can still be observed, if the estimation method overestimates them. For the Headlamp part (figure 9) the maximum thickness value was taken from the physical measurements. 


\subsection{Improvement over previous method}

The top rows of figures 6 and 7 present, for a Door Handle and B-Pillar Trim, respectively, the estimated thickness obtained with the method from [5] (sub figures 6(a) and 7(a)) and the newly proposed improved method (sub figures $6(\mathrm{~b})$ and $7(\mathrm{~b})$ ). The bottom rows of these figures present the difference (absolute value) between the estimated thickness and the thickness obtained by visual inspection of the CAD files for each element of the parts' midplane meshes. Direct comparisons of figure 6(c) with 6(d) and of figure 7(c) with $7(\mathrm{~d})$ clearly show that:

- the improved thickness estimation method is much more effective than that achieved by the previous method, with the difference to the visual inspection thickness being much closer to zero - note the predominance of blue regions, which indicate difference values close to zero;

- thickness over-estimation near ribs, a typical problem in the previous method, is now solved.
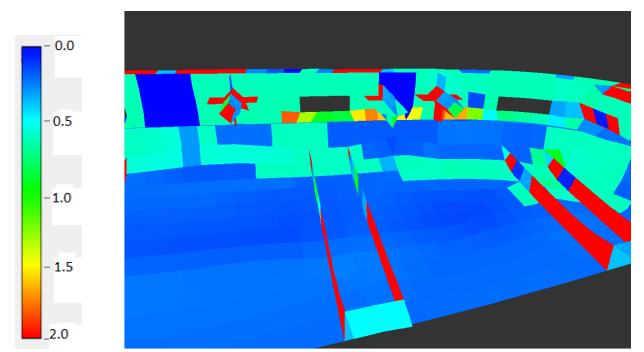

(a) Thickness from [5].
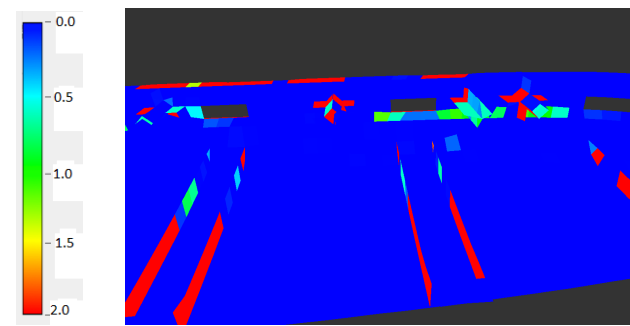

(c) Difference between 6(a) and visual inspection
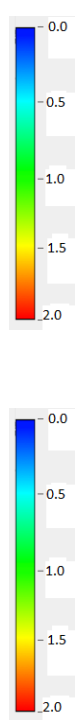

(d) Difference between 6(b) and vi-
sual inspection

Figure 6: The Door Handle part - pseudo color maps for thickness estimates with both methods (top row) and difference to visual inspection measurements (bottom row) (normalized thickness expressed in $\mathrm{mm} / \mathrm{mm}$ ).

In order to quantify the improvements obtained with the newly proposed approach, table 1 presents the RMSE for each thickness estimation method, comparing the respective estimates with values resulting from visual inspection. Gain is computed as the ratio between the RMSE of the previous method [5] and the method proposed in this 


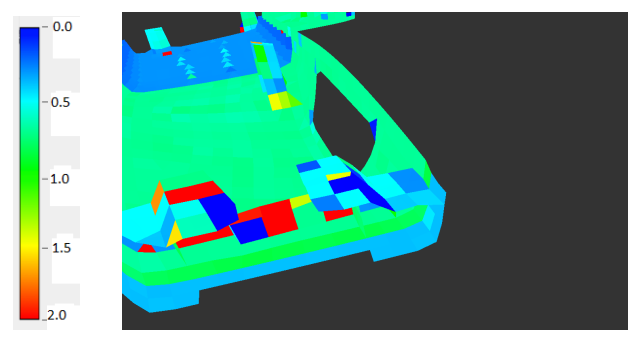

(a) Thickness from [5].
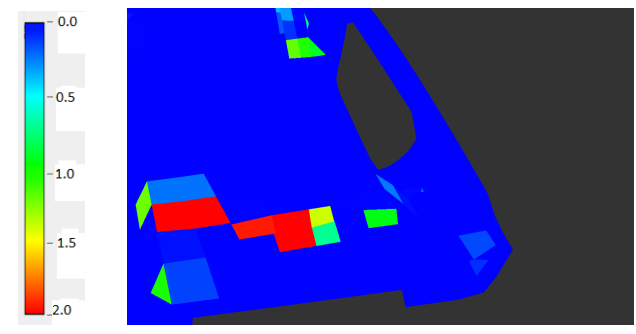

(c) Difference between 7(a) and visual inspection
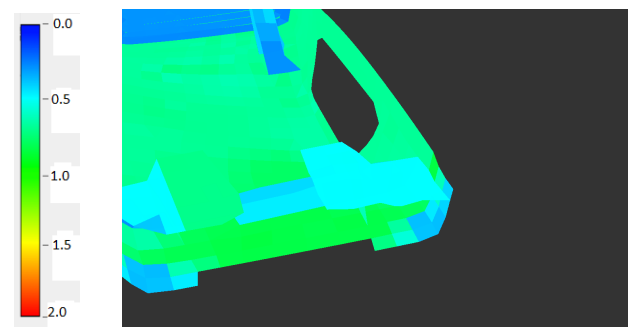

(b) Improved estimation.
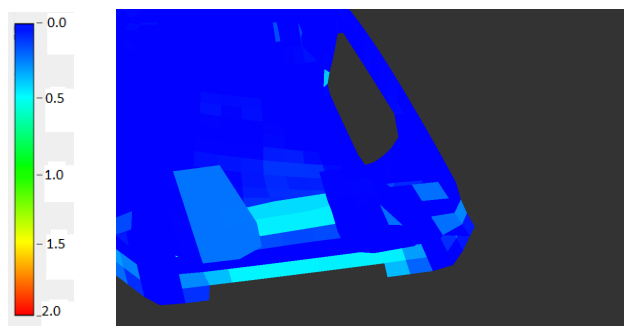

(d) Difference between 7(b) and visual inspection

Figure 7: The B-Pillar Trim part - pseudo color maps for thickness estimates with both methods (top row) and difference to visual inspection measurements (bottom row) (normalized thickness expressed in $\mathrm{mm} / \mathrm{mm}$ ).

paper.

\begin{tabular}{l|r|r|r} 
Region & \multicolumn{1}{|c}{$\begin{array}{c}\text { Door Handle } \\
{[5]} \\
(\mathrm{mm} / \mathrm{mm})\end{array}$} & $\begin{array}{c}\text { Proposed } \\
(\mathrm{mm} / \mathrm{mm})\end{array}$ & \multicolumn{1}{c}{ Gain } \\
\hline Boss 1 & 0.576 & 0.261 & 2.212 \\
Boss 2 & 0.493 & 0.313 & 1.574 \\
Rib & 69.990 & 0.445 & 157.448 \\
Boss 3 & 48.723 & 3.315 & 14.696 \\
\hline
\end{tabular}

\begin{tabular}{l|r|r|r} 
Region & \multicolumn{1}{c}{$\begin{array}{c}\text { B-Pillar Trim } \\
{[5]} \\
(\mathrm{mm} / \mathrm{mm})\end{array}$} & $\begin{array}{c}\text { Proposed } \\
(\mathrm{mm} / \mathrm{mm})\end{array}$ & \multicolumn{1}{|c}{ Gain } \\
\hline Boss 1 & 0.057 & 0.056 & 1.018 \\
Boss 2 & 13.661 & 1.361 & 10.039 \\
Boss 3 & 2.600 & 0.474 & 5.483 \\
Boss 4 & 1.619 & 0.262 & 6.171 \\
\hline
\end{tabular}

Table 1: RMSE comparison of thickness estimates with results from visual inspection (normalized values)

The RMSE of the proposed improved approach is much lower than that obtained with the previous approach, except for the Boss 1 region of the B-Pillar trim part where the original estimate already presents a very low RMSE. Thickness estimates are much more accurate, specially near ribs and highly embossed areas. RMSE gain often reaches two digits, and, for the Rib of the Door Handle part, goes up to 157; the gain is low only when the original error was already low. These results allow us to conclude that the improved thickness estimation method generates estimates which are very similar to those that would be obtained by having an human operator visually inspecting the parts' CAD files and from there measuring thickness for each midplane mesh element, 
which is an extremely time consuming and error-prone process.

Figure 8 depicts the thickness estimates obtained with the two alternative methods and visual inspection, for different regions of the Door Handle part. Results are presented for the different regions of the part where visual inspection was performed. The improved estimate (green line) is much smoother and follows the values obtained from visual inspection (blue line) much closer than estimates obtained with the previous method (red line).

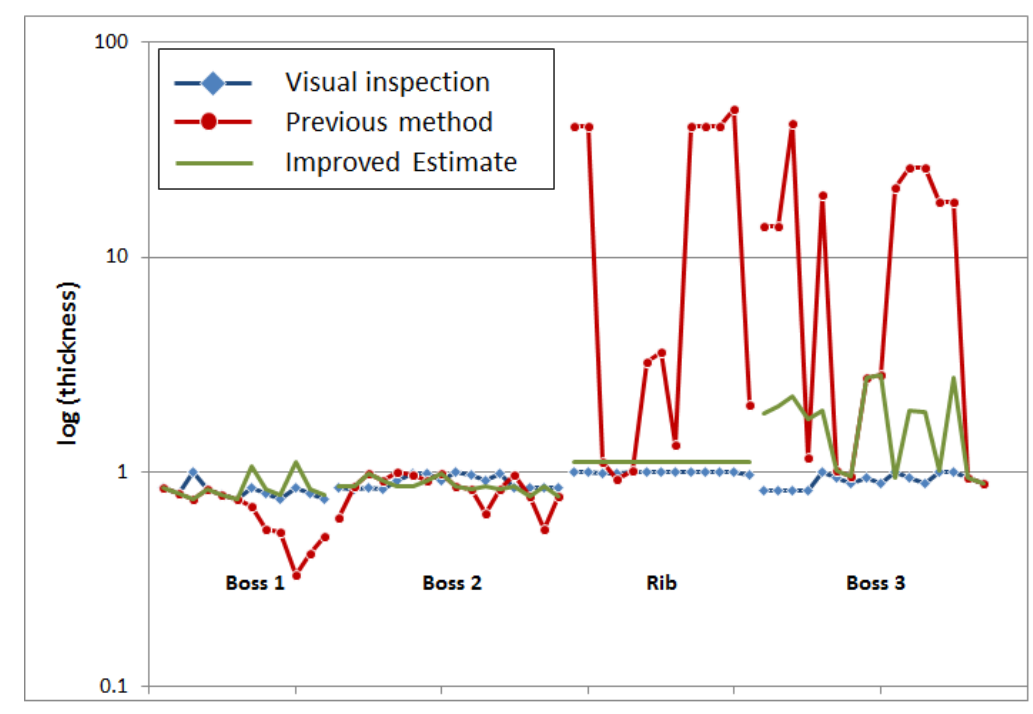

Figure 8: Normalized thickness estimates obtained with both methods and visual inspection for the Door Handle part(log vertical axis).

\subsection{Comparison with Thickness Obtained by Physical Inspection}

Automated and precise ways to extract local thickness distribution from the CAD files are important to prevent limiting the precision (and thus, the potential benefit) of crash simulations. Performing measurements by physical inspection requires significant amount of manual labor and is prone to several errors. In fact, different operators subjectively extract different measurements in the same location of the same part. Even measurements by the same operator in different days will exhibit some variation. In addition, as stated in section 1, crash simulations are usually performed before actual production of real physical parts, which prevents physical inspection. Nevertheless, in order to further validate the proposed thickness estimate method, we requested two human operators to independently perform a set of thickness measurements along a real part.

Figure 9 compares the estimated thickness using our method and the average of thickness values obtained by physical inspection by the two operators, along a set of 138 points 
of the Headlamp part, as described in subsection 4.1. The estimated thickness curve (black line) clearly follows the physically measured thickness (red line), even though there is a slight tendency to underestimate it. RMSE is equal to 0.07, which confirms the conclusions drawn on the previous subsection and reinforce our confidence that the proposed approach estimates thickness with high accuracy.

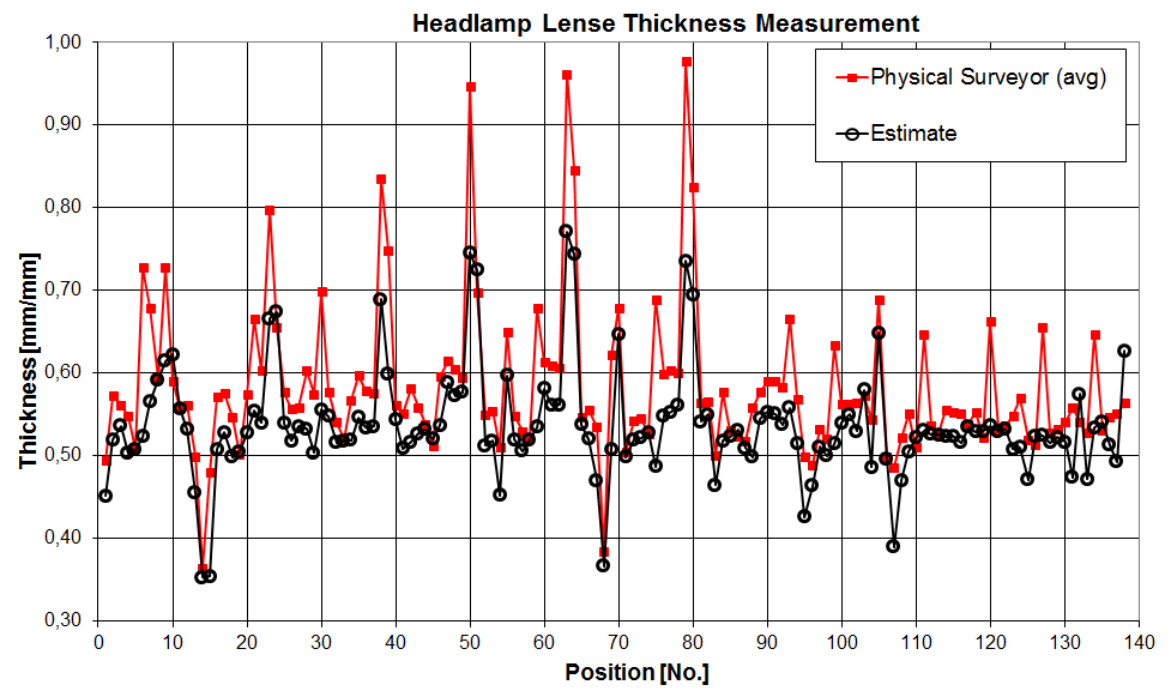

Figure 9: Comparison of estimated thickness versus measurements obtained by physical inspection of the Headlamp part.

\subsection{Model Validation through Experimental Crash Tests}

To show the effectiveness of the proposed approach with respect to simulation quality increase, crash simulations have been performed with the standard procedure (manual classification of the parts into distinct areas with nominal thickness) and the advanced procedure (element wise thickness estimations obtained with the improved method). Both approaches are directly compared with related dynamic physical component tests performed at the Ford Motor Company. Note that whereas results presented in the previous subsections verified the accuracy of thickness estimations, results presented here assess the impact this added accuracy has on the crash simulations themselves. This assessment is performed by comparing the simulation results with actual physical tests.

For this assessment a pedestrian protection load case has been chosen, which is one of the various crash load cases that are usually optimized using CAE in the development process. The accuracy of the method is very critical because legislative boundary conditions must be met, e.g. maximum forces, accelerations, bending angles. If the simulation quality is not accurate enough the engineer could be directed in the wrong 
direction regarding part design which, in worst case, will lead to not complying with legislation, therefore making expensive re-construction and re-tests of the specific part inevitable.

A deformable impactor representing a human upper leg is impacted against a vehicle front in the area of the headlamp, as shown in figure 10(a). The headlamp has to absorb enough energy to keep the forces on the human being as low as possible to avoid critical injuries. Due to the fact that the headlamp is made of different injection molded plastic components, which show significant thickness variations, the deformation and fracture is highly dependent on local thickness distributions.

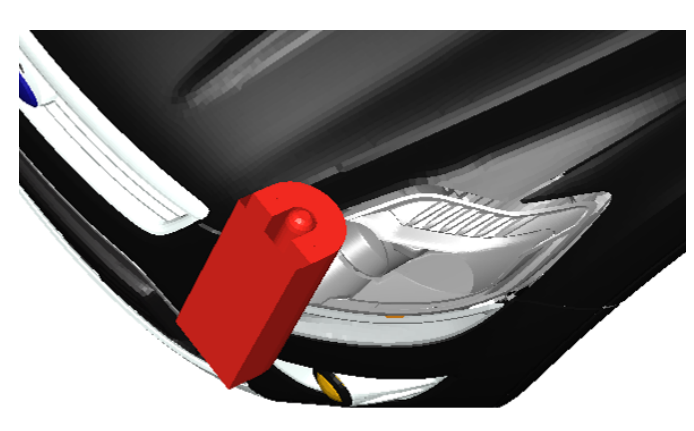

(a) Pedestrian protection test setup.

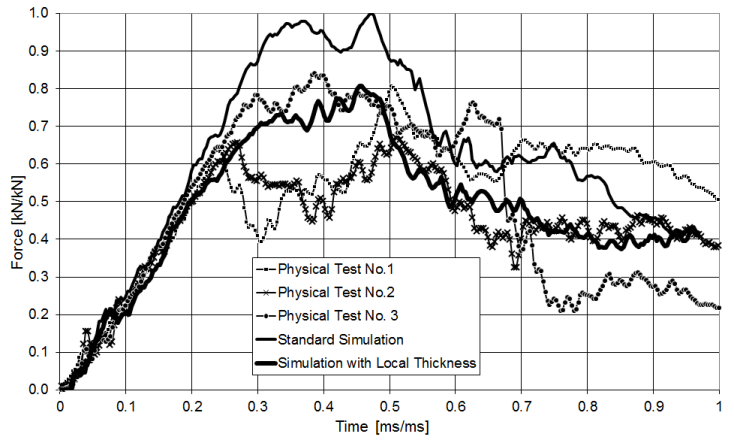

(b) Comparison of physical tests and simulations.

Figure 10: Crash simulation load case: upper leg impact on the headlamp.

The dynamic impact simulations have been performed at Ford Motor Company with a front subsystem model. Within this model all components of the frontend are modeled in detail to correctly consider deformation and loading of all involved components. This is needed to correctly predict the pedestrian protection behavior of the vehicle. Crash simulation software RADIOSS Explicit V11 using a user defined material model has been used for these dynamic simulations. The visco-elastic, visco-plastic material model, explicitly developed for thermoplastic materials, takes into account the asymmetric yield and hardening as well as the stress triaxiality to determine fracture. The mid-plane mesh consists of hourglass stabilized four and three node shell elements with an element length of around $3 \mathrm{~mm}$ representing the parts geometry. This approach is state of the art within automotive engineering.

Figure 10(b) depicts the validation results in detail. It is obvious that the high dynamic physical tests show some variability due to several factors (complex load case, complex impactor and several complex components involved in the impact event). However, it is clear from this figure that the explicit dynamic crash simulation using local thickness mapping predicts the expected mechanical part behavior with considerably higher accuracy than the standard thickness estimation method resulting in a good force-deflection prediction. The significant improvement of the new local thickness estimation method over the standard simulation approach is due to the availability of local thickness infor- 
mation in the critical impact area of the headlamp, e.g. lens attachment to housing, lens edges and features within the lens and housing. These results also show that an advanced material model with a highly sophisticated fracture module can only be predictive if the correct local thickness is taken into account.

While this work is focused on crash (e.g., explicit simulations) the proposed method would be equally useful for other modes like Stiffness, Noise Vibration Harshness (NVH) and Durability (e.g., implicit simulations) and materials that are injection molded or casted, e.g. thermoplastics, aluminum, magnesium. Therefore this approach will give the possibility of significantly enhance simulation quality, leading to the overall goal of zero prototype strategy to reduce development time and resources. This is why Ford Motor Company is already using this methodology within product development and is analyzing the overall potential of this method for all areas of simulation.

\subsection{Results' Synthesis}

The results presented throughout section 4 demonstrate that the newly proposed thickness estimation method produces estimates that:

i) are more accurate than those produced by the previous existing method;

ii) impact significantly on the accuracy of crash simulations compared to standard approaches, by making available local thickness information.

In subsection 4.2 the new method has been compared with the previously published approach [5] and results compared by using as reference thickness values obtained by visual inspection of the CAD files. It has been shown that the improved thickness estimation method is very effective, presenting RMSE gains over the previous method that often reach two digits. Additionally, thickness over-estimation near ribs, a typical problem in the previous method, is now solved.

In order to further validate the effectiveness of the proposed method its estimates are compared with measurements obtained by physical inspection of a real part, as measured by two human operators - subsection 4.3. Results show that even though there is a slight tendency to underestimate thickness, the estimates are very accurate, with a RMSE of 0.07 for this particular part.

The results obtained by comparing thickness estimates with values obtained both by visual and physical inspection show that the proposed method is highly reliable.

Finally, in subsection 4.4 the impact of the availability of local thickness information on the accuracy of the actual crash simulations is assessed. The crash simulation results obtained with local information and those obtained with the standard method (nominal thicknesses) are compared with results from dynamic physical component tests performed at the Ford Motor Company. It has been shown that dynamic crash simulation using local thickness mapping predicts the expected mechanical part behavior 
with considerably higher accuracy than the standard nominal thickness method, thus demonstrating the relevance of local thickness information and the suitability of the proposed approach to estimate it.

\section{Conclusion}

The standard procedure in engineering practice for crash simulations is assigning a nominal thickness to distinct areas in parts. However, thickness changes significantly along the part, and it is not feasible to adequately assign that thickness to the FEM model by hand. Moreover, even measuring the thickness in a real part is subjective and prone to operator error or bias, as we have also demonstrated. The method we propose in this paper for estimating local thickness exhibits a substantial performance improvement to a previous version, by automatically adopting the best of two different estimates and also resorting to thickness propagation. It was demonstrated that the method is now able to adequately reproduce the thickness distribution in the part, based on CAD data.

As our results clearly show, the proposed local thickness estimation method enables obtaining more accurate prediction of the crash behavior of engineering parts. The simulations performed with a local thickness distribution, using the proposed method, exhibit a much better agreement with dynamic physical component tests. Having more reliable simulation results can affect the entire development cycle, with special incidence at the the product development stage and enabling the adoption of zero-prototyping development processes. This is particularly important for very complex parts and those with demanding specifications, as is usually the case in automotive, aeronautics, defense, and other areas of application.

In summary, this work represents a significant contribution towards improved simulations of the mechanical behavior of engineering parts, and can have a substantial impact on engineering practice in industry as is the case at Ford Motor Company, which is already using the proposed approach.

\section{Acknowledgements}

The authors would like to thank the FCT - Fundação para a Ciência e a Tecnologia (Portuguese Foundation for Science and Technology) through projects PEst-C/CTM/LA0025/2013 and PEst-OE/EEI/UI0752/2011. 


\section{References}

[1] A. Appel. Some techniques for machine rendering of solids. In AFIPS Conferences, number 32, pages $37-45,1968$.

[2] D. Carstêa. Semiautomatic Generation of Database in Finite Element Methods. International Journal of Mathematical Models and Methods in Applied Sciences, 1(2):76, 2007.

[3] M. de Berg. Computational Geometry: Algorithms and Applications. Springer, 2008 .

[4] M. H. DeGroot. Probability and Statistics. Addison-Wesley, Reading, Massachusetts, 1980.

[5] V. Ferreira, L.P. Santos, M. Franzen, O.O. Ghouati, and R. Simoes. Estimating Local Part Thickness in Midplane Meshes for Finite Element Analysis. International Journal of Mathematics and Computers In Simulation, 5:69, 2011.

[6] R. Gal, A. Shamir and D. Cohen-Or. Pose-Oblivious Shape Signature. IEEE Transactions On Visualization And Computer Graphics, 13(2):261-271, 2007.

[7] H. R. Karimi, W. Pawlus and K. G. Robbersmyr. Signal reconstruction, modeling and simulation of a vehicle full-scale crash test based on Morlet wavelets. Neurocomputing, 93(15):88-99, 2012.

[8] B. Kirk, J. W. Peterson, R. H. Stogner, and G. F. Carey. libMesh: A C++ Library for Parallel Adaptive Mesh Refinement/Coarsening Simulations. Engineering with Computers, 22(3):237 - 254, 2006.

[9] X. Rolland-Nevière, G. Doërr and P. Alliez. Robust diameter-based thickness estimation of 3D objects. Graphical Models, 75:279 - 296, 2013.

[10] W. Pawlus, H. R. Karimi and K. G. Robbersmyr. Reconstruction and simulation of the vehicle to road safety barrier oblique collision based on the Levenberg-Marquardt algorithm. International Journal of Crashworthiness, 17(6):676-692, 2012.

[11] W. Pawlus, H. R. Karimi and K. G. Robbersmyr. Data-based modeling of vehicle collisions by nonlinear autoregressive model and feedforward neural network. Information Sciences, 235(20):65-79, 2013.

[12] G. Strang and G. Fix. An Analysis of The Finite Element Method. Prentice Hall, 1973.

[13] E. De Sturler, G. H. Paulino, and S. Wang. Topology optimization with adaptive mesh refinement. In 6th International Conference on Computation of Shell and Spatial Structures, Ithaca, New York, USA, 2008. 
[14] M. Vandewettering, E. Haines, E. John Kalenda, R. Parent, S. Uselton, and "Zap". Point on Polygon, One More Time .... Ray Tracing News, 1990.

[15] T. Whitted. An Improved Illumination Model for Shaded Display. ACM Communications, 23(6):343-349, 1980.

[16] T.Y. Yang. Finite Element Structural Analysis. Prentice-Hall (Englewood Cliffs, N.J.), 1986.

[17] L. Zhao, W. Pawlus, H. R. Karimi and K. G. Robbersmyr. Data-Based Modeling of Vehicle Crash Using Adaptive Neural-Fuzzy Inference System. IEEE/ASME Transactions on Mechatronics, 2013. 